Revue internationale de l'économie sociale

Recma

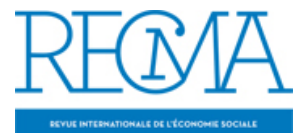

\title{
Les prix de l'Addes 2013, rapport du jury
}

\section{Maurice Parodi}

Numéro 331, janvier 2014

URI : https://id.erudit.org/iderudit/1023488ar

DOI : https://doi.org/10.7202/1023488ar

Aller au sommaire du numéro

Éditeur(s)

Association Recma

ISSN

1626-1682 (imprimé)

2261-2599 (numérique)

Découvrir la revue

Citer cet article

Parodi, M. (2014). Les prix de l'Addes 2013, rapport du jury. Revue

internationale de l'économie sociale, (331), 108-117.

https://doi.org/10.7202/1023488ar d'utilisation que vous pouvez consulter en ligne.

https://apropos.erudit.org/fr/usagers/politique-dutilisation/ 


\title{
LES PRIX DE L'ADDES 2013, RAPPORT DU JURY
}

\author{
par Maurice Parodi*
}

* Président du jury Addes.

La constitution et le développement d'une communauté scientifique ayant pour objet l'économie sociale ont été et demeurent l'un des objectifs fondamentaux de l'Addes, Association pour le développement de la documentation sur l'économie sociale. Le moyen essentiel pour atteindre cet objectif a été la création des prix de thèse et de mémoire attribués à de jeunes chercheurs lors de chaque colloque depuis 1986, grâce à la générosité de la Fondation Crédit coopératif, laquelle apporte aussi un soutien sans faille à la Recma.

La riche cuvée 2013 de thèses et de mémoires (niveau master 1 et 2) témoigne du succès de l'entreprise vingt-sept ans après cette création, à la fois pour le nombre de thèses et de mémoires qui ont concouru pour le prix 2013, par la qualité scientifique de ces travaux, par la diversité des thématiques choisies par les jeunes chercheurs ou encore par la diversité géographique des universités et des équipes ou centres de recherche doctorale qui ont soutenu ces travaux et, enfin, par la palette des disciplines scientifiques mobilisées. Le tableau 1 (en page suivante) synthétise cette diversité et cette richesse. L'annexe présente la liste intégrale des thèses et des mémoires soumis au jury de l'Addes, leur nature disciplinaire, leur objet (intitulé) et les universités d'origine. Tous ces travaux sont consultables au centre de documentation du Crédit coopératif ${ }^{(1)}$.

(1) 12, boulevard Pesaro, CS 10002, 92024 Nanterre cedex. Contact: Véronique Larosée, tél. 0147248562 , mél. veronique.larosee@ credit-cooperatif.coop.

\section{RAPPROCHER LES ACTEURS ET LES CHERCHEURS}

Ce survol des terroirs d'origine contrôlés (universités...) et de la diversité des cépages (disciplines scientifiques) qui ont nourri les thèses et les mémoires du cru 2013 montre bien que l'Addes et la Fondation Crédit coopératif ont efficacement contribué - avec la Recma, d'autres fondations de l'économie sociale, le Comité national de liaison des activités mutualistes, coopératives et associatives (Cnlamca) en son temps, le Réseau inter-universitaire de l'économie sociale et solidaire (Riuess), l'Association d'économie sociale et les institutions publiques comme la Dies, la Mire... - à la création et au développement d'une communauté scientifique pour l'objet de recherche "économie sociale".

En appui (direction de recherche, tutorat...) des trente-quatre jeunes chercheurs qui concouraient cette année pour décrocher les prix de l'Addes, on doit logiquement dénombrer, pour les « thésards ", autant de directeurs de recherche ou d'équipes doctorales et, pour les mémoires en particulier, autant de tuteurs de stage ou de cadres ou dirigeants de structures 


\section{Tableau 1}

\section{Présentation synthétique de la diversité des thèses et mémoires}

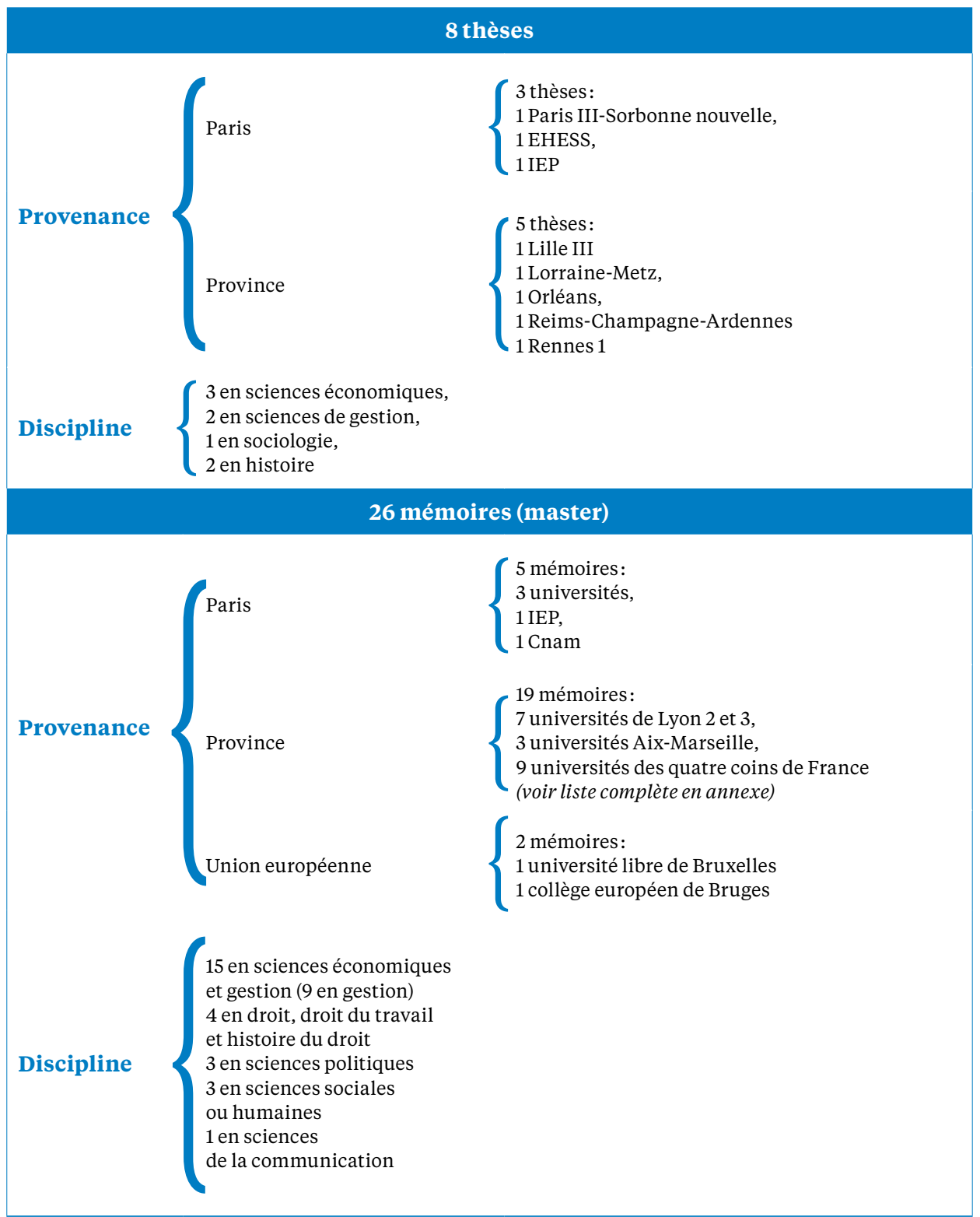

de l'économie sociale qui ont accueilli, suivi, conseillé les apprentis chercheurs sur les terrains diversifiés d'application ou d'expérimentation de leurs travaux. Par là même, on peut voir que l'une des ambitions de l'Addes, qui était de réduire la distance entre chercheurs et praticiens de l'économie sociale ou entre « les auteurs et les acteurs ", selon les termes d'Henri Desroche, n'était pas complètement utopique. 


\section{LE PRIX DE THĖSE}

Le prix de thèse a été décerné à Jessica Dos Santos, «L'utopie en héritage: la Société du Familistère de Guise, de la mort de Jean-Baptiste Godin à la dissolution de l'association (1888-1968) » (thèse d'histoire sous la direction de Jean-François Eck, université de Lille 3).

Cette thèse, incontournable pour tous ceux qui s'intéressent encore à l'ambition sociétale ou politique du projet coopératif (ou de la « république coopérative » selon Charles Gide ou Ernest Poisson), déborde très largement de la simple recherche de type monographique ou micro-économique. Elle explore le cœur même du projet utopique de J.-B. Godin, ses paradoxes, ses contradictions, ses défis, ses enjeux et ses spécificités méritoires tels qu’ils se révèlent tout au long de l'histoire mouvementée de la société et de l'entreprise du Familistère, de sa fondation, en 1888, à sa liquidation, en 1968.

L'originalité et l'intérêt spécifique de cette thèse ne se trouvent pas dans une nouvelle exploration du modèle utopique de la Société du Familistère de Guise, mais bien dans la confrontation rigoureuse du modèle aux "événements démesurés » qui ont marqué l'histoire de l'association et de l'entreprise ellemême (deux guerres mondiales, la grande crise des années 30, l'occupation de la France par les troupes allemandes, la reconstruction, l'ouverture et la mondialisation du marché...). Ces événements, les défis qui ont été soulevés et les conflits qui en ont résulté aussi bien entre la société et son environnement local, national ou international qu'entre les parties prenantes de l'entreprise elle-même (direction, associés, salariés...) ont entraîné bien des déviations, voire des changements de cap par rapport aux ambitions du projet originel. Mais peut-on parler de son échec final? Les grands acteurs de l'économie sociale d'aujourd'hui ou du " projet coopératif » ne peuvent-ils puiser encore dans l'histoire de la Société du Familistère de Guise quelque aspiration nouvelle ou simplement renouvelée? Oui, sans doute, s'ils croient au patrimoine de l'économie sociale. Mais cela renvoie à une double exclamation d'Henri Desroche (à la fois admirative et un peu désabusée): «Ô (oh!) Patrimoines. Alerte à une désertification» (Asscod, $\mathrm{n}^{\circ} 83$, janvier-mars 1988).

\section{Les autres thèses}

Si le jury n'a pas primé la thèse de Florence Gallois, d'excellente facture universitaire, «Une approche régulationniste des mutations de la configuration institutionnelle française des services à la personne " (thèse de sciences économiques, sous la direction de Martino Nieddu, université de Reims Champagne-Ardenne), c'est parce qu'elle avait déjà été distinguée par le prix de l'Association d'économie sociale.

Enfin, grâce à la générosité de la Fondation Crédit coopératif, le jury a pu décerner deux prix spéciaux à deux autres candidats, "nominés " au premier tour pour la qualité exceptionnelle de leur thèse. C'est dire l'embarras du jury pour départager candidates et candidats du millésime 2013.

\section{$1^{\text {er }}$ prix spécial}

L’un des prix spéciaux a été décerné à un jeune docteur italien, Niccoló Mignemi, pour sa thèse d'histoire et de civilisations soutenue en France, 
à l'EHESS (sous la direction de Gérard Beaur et la codirection d'Angelo Moióli, de l'université catholique de Milan): "Coopérer pour travailler, coopérer pour exploiter la terre. Itinéraires comparés des coopératives agricoles en Italie et en France dans la première moitié du $\mathrm{XX}^{\mathrm{e}}$ siècle ». Des itinéraires comparés mais peu comparables, car l'histoire des coopératives agricoles italiennes s'enracine dans la question sociale des campagnes et des paysans pauvres en lutte contre l'exploitation par les propriétaires latifundiaires du sud de l'Italie. Comme point de départ de sa recherche, l'auteur choisit donc un des hauts lieux de la tradition coopérative italienne, la Sicile (l'emblématique terre du Guépard). Aux fermages collectifs vont succéder des coopératives de travail et d'affermage. Tradition qui se poursuit aujourd'hui, à l'occasion de la redistribution des grands domaines accumulés par la mafia (économie du crime organisé) aux paysans pauvres.

L'histoire des coopératives agricoles françaises est tout autre, comme le savent bien les fidèles lecteurs de la Recma. Elle est très liée à la politique d'encadrement agricole de la France (sous Méline...) dans le contexte de la grande crise du tournant du siècle, puis du premier conflit mondial, de la grande crise économique de l'entre-deux-guerres, de la Seconde Guerre mondiale et de l'après-guerre. Mais la thèse s'arrête en 1950, c'est-à-dire avant «la grande transformation » de l'agriculture française des années 50 et 60 , sous Edgard Pisani. Cela débouche sur le système coopératif agricole diversifié et complémentaire que nous connaissons encore aujourd'hui. Il s'agit toujours, au moins dans les régions où dominent productions viticoles ou fruitières, élevage, laiterie et fromagerie, d'exploiter la terre, d'en transformer les produits, de les commercialiser sur des marchés de plus en plus ouverts, d'en moderniser et d'en structurer les exploitations familiales et de les protéger contre la concurrence exacerbée des marchés et la pression des monopoles. Outre la valeur scientifique de cette monumentale recherche d'histoire économique, dans la tradition d'un F. Braudel, l'intérêt de cette thèse, pour un prix de l'Addes, réside bien dans la confrontation de ces deux histoires de la coopération agricole dans des mondes paysans et agraires si différents. En effet, " si la formule universelle de la coopération est accessible à tout le monde (pour les petits et pour les grands), pour les plus grands et les plus puissants la coopération constitue une solution parmi d'autres, alors que pour les paysans (de Sicile comme pour les exploitants familiaux français), elle constitue souvent la seule opportunité sociétaire praticable pour sortir de l'isolement des stratégies individuelles et renforcer leur pouvoir, sans être obligés de fournir des capitaux et des garanties dont ils ne peuvent pas disposer». En bref, pour eux, la coopération reste la fille aînée de la nécessité et, grâce à elle, la ruse des paysans peut encore accommoder la contrainte des choses...

\section{$2^{\mathrm{e}}$ prix spécial}

Le second prix spécial (par ordre alphabétique des auteurs) a été décerné à Charles Sellen pour sa thèse de sciences économiques soutenue à l'IEP de Paris (sous la direction de Patrick A. Messerlin), « Philanthropie et économie: essais autour de la générosité ». Un travail d'investigation passionnant dans les formes multiples que peuvent revêtir la philanthropie et la générosité des individus, des organisations, des entreprises privées ou même 
des organismes publics (la CDC, par exemple) qui entendent contribuer au bien public par leurs subsides et, mieux encore, par «leur capacitéà mettreen relation des individus qui s'ignorent », parfois aux deux extrémités de la terre. Un chapitre entier, non dénué d'esprit critique, s’intéresse au rôle des plus grandes fondations américaines, pour l'essentiel sous l'influence du "paradigme culturel particulier héritéde l'éthique protestante »(Max Weber), sur la scène du développement international et de «biens publics mondiaux». Le dernier chapitre s'attache logiquement à décrypter "le mystère de l'altruisme dans l'économie ", aussi bien dans la théorie néoclassique que dans le fonctionnement et la structure du système économique dominant. De quelle nature et quel est le « sens » d'une philanthropie au sein même du « système du capitalisme » et de son paradigme propre?

\section{LE PRIX DE MÉMOIRE}

Le prix de mémoire a été attribué à un jeune chercheur, Alexeï Tabet, pour son mémoire d'histoire (de master 1 , donc en quelque sorte pour son premier essai!) intitulé " L'invention du tiers secteur: les commencements de l'économie sociale et solidaire ", soutenu à l'IEP de Paris (sous la direction de Marc Lazar).

Le titre même du mémoire est un clin d'œil manifeste à l'ouvrage de référence d'André Gueslin sur l'invention de l'économie sociale par les grands auteurs et acteurs du XIX ${ }^{\mathrm{e}}$ siècle, dont Alexeї Tabet s'est fortement inspiré. Mais son travail s'applique particulièrement à repérer les auteurs et les acteurs, dont Jacques Delors, Jocelyne Gaudin ou les éclaireurs d'une « troisième voie » comme François Bloch-Lainé, Pierre Rosanvallon, Ivan Illich, Patrick Viveret..., qui au cours des quarante dernières années, en France, se sont attachés à promouvoir le concept de tiers secteur, qui a précédé la réémergence de celui d'économie sociale à la fin des années 70 , à l'initiative avérée d'Henri Desroche et avec l'appui de Jacques Moreau, du Cnlamca et $\mathrm{du}$ Centre interdisciplinaire de recherche et d'information sur les entreprises collectives (Ciriec).

Là encore, il ne faut pas perdre la trace des cinq autres "nominés » du premier tour, ni même celle de la plupart des auteurs de mémoire, car leurs travaux peuvent être d'une grande utilité pour les acteurs de l'ESS ou pour les entrepreneurs sociaux, en fonction de leur secteur d'activité et de leur projet spécifique. C'est la raison pour laquelle nous les invitons à explorer la liste complète de ces mémoires en annexe.

\section{Les « nominés "}

S’agissant des cinq autres nominés, trois avaient déjà été distingués par le prix de la Fondation du Crédit mutuel, ce qui nous a permis de les retirer sans trop de remord de la sélection au deuxième tour. Il s'agit de: Pierre Francoual, pour "Les travailleurs dans la société coopérative ouvrière de production " (master en droit du travail, soutenu à l'université Toulouse-Capitole); Camille Meyer, pour « Les finances solidaires comme biens communs durables: étude de cas de la banque communautaire de développement Palmas " (master en sciences de la population et du développement, université libre de Bruxelles); 
et Loïc Seeberger, pour « Essai sur le droit coopératif français, de ses

(2) Cestravauxsontconsultables sur www.recma/node/3160. origines à la $\mathrm{V}^{\mathrm{e}}$ République: entre pratiques et normes juridiques » (master en histoire du droit, université Montpellier I) (2).

Deux autres mémoires avaient été retenus au premier tour par plusieurs membres du jury et méritent, au moins, d'être cités: Sylvain Celle pour « Le mouvement coopératif dans le socialisme de Jean Jaurès et Marcel Mauss » (master en économie appliquée, université Lille 1) et Carine Hartmann pour « Les mutations du secteur culturel: vers une économie plus créative, solidaire et durable » (master en ingénierie de projets en ESS, université de Strasbourg).

Pour ceux qui suivent de près l'évolution lente mais réelle de la reconnaissance institutionnelle des spécificités des formes organisationnelles et du "système de règles» (C. Vienney) des entreprises et organisations de l'économie sociale (coopératives, mutuelles, associations, fondations) ou des «entreprises sociales » (selon l'acception plus large de la commission de l'Union européenne), nous recommandons vivement, in fine, le mémoire d'Ugo Bessière, soutenu au collège d'Europe de Bruges: « Quelle place pour les modèles alternatifs dans l'Union européenne? ». 


\section{Annexe}

\section{Travaux reçus pour le Prix 2013 de l'Addes}

\section{Thèses}

\begin{tabular}{|c|c|c|c|c|}
\hline Nom & Prénom & Sujet & Discipline & Université \\
\hline Ambroisine & Joël & $\begin{array}{l}\text { "Les relations professionnelles } \\
\text { dans le milieu associatif: } \\
\text { analyse comparative } \\
\text { entre la France, l’Espagne } \\
\text { et le Royaume-Uni » }\end{array}$ & $\begin{array}{l}\text { Sciences } \\
\text { économiques }\end{array}$ & $\begin{array}{l}\text { Paris III } \\
\text { Sorbonne- } \\
\text { Nouvelle }\end{array}$ \\
\hline Dos Santos & Jessica & $\begin{array}{l}\text { "L'utopie en héritage: } \\
\text { la Société } \\
\text { du Familistère de Guise, } \\
\text { de la mort de J.-B. Godin } \\
\text { à la dissolution } \\
\text { de l'association (1888-1968)» }\end{array}$ & Histoire & Lille \\
\hline Gallois & Florence & $\begin{array}{l}\text { "Une approche régulationniste } \\
\text { des mutations de la configuration } \\
\text { institutionnelle française } \\
\text { des services à la personne " }\end{array}$ & $\begin{array}{l}\text { Sciences } \\
\text { économiques }\end{array}$ & $\begin{array}{l}\text { Reims- } \\
\text { Champagne- } \\
\text { Ardenne }\end{array}$ \\
\hline Mignemi & Niccoló & $\begin{array}{l}\text { "Coopérer pour travailler la terre, } \\
\text { coopérer pour exploiter la terre. } \\
\text { Itinéraires comparés des coopératives } \\
\text { agricoles en Italie et en France } \\
\text { dans la première moitié du xx } x^{\mathrm{e}} \text { siècle " }\end{array}$ & Histoire & EHESS \\
\hline $\begin{array}{l}\text { Morales } \\
\text { La Mura }\end{array}$ & Quidora & $\begin{array}{l}\text { "Contre-pouvoir, technicité } \\
\text { et action associative» }\end{array}$ & Sociologie & Lorraine \\
\hline $\begin{array}{l}\text { Ould } \\
\text { Moulaye } \\
\text { Ismaïl }\end{array}$ & $\begin{array}{l}\text { Moulaye } \\
\text { Abdelkader }\end{array}$ & $\begin{array}{l}\text { "Valeurs religieuses et critères } \\
\text { de choix d'une banque } \\
\text { en relation avec la création } \\
\text { des banques islamiques en France» }\end{array}$ & $\begin{array}{l}\text { Sciences } \\
\text { de gestion }\end{array}$ & Rennes I \\
\hline Saunier & $\begin{array}{l}\text { Paul- } \\
\text { Laurent }\end{array}$ & $\begin{array}{l}\text { "Qualité et contrôle dans les services: } \\
\text { le cas des services à la personne" }\end{array}$ & $\begin{array}{l}\text { Sciences de } \\
\text { gestion }\end{array}$ & Orléans \\
\hline Sellen & Charles & $\begin{array}{l}\text { "Philanthropie et économie: } \\
\text { essais autour de la générosité » }\end{array}$ & $\begin{array}{l}\text { Sciences } \\
\text { économiques }\end{array}$ & IEP Paris \\
\hline
\end{tabular}


Mémoires

\begin{tabular}{|c|c|c|c|c|}
\hline Nom & Prénom & Sujet & Discipline & Université \\
\hline Ardoin & Perrine & $\begin{array}{l}\text { "Quels sont les enjeux et défis } \\
\text { que doit relever une entreprise } \\
\text { implantée au Sénégal } \\
\text { à travers sa RS sociétale?» }\end{array}$ & $\begin{array}{l}\text { Master } 2 \\
\text { ESS }\end{array}$ & Lyon 2 \\
\hline Balaine & Alexis & $\begin{array}{l}\text { "Le knowledge management, } \\
\text { une solution pour transmettre les } \\
\text { connaissances" }\end{array}$ & $\begin{array}{l}\text { Master } 2 \\
\text { Management } \\
\text { de l'économie } \\
\text { solidaire }\end{array}$ & $\begin{array}{l}\text { IUP } \\
\text { management } \\
\text { social et santé, } \\
\text { Caen }\end{array}$ \\
\hline Baux & Angélique & $\begin{array}{l}\text { "L'impact des différents modèles } \\
\text { de gouvernance sur les sociétés } \\
\text { d'assurance mutuelles: d'une mort } \\
\text { annoncée à la réaffirmation } \\
\text { du modèle mutualiste» }\end{array}$ & MBA & Cnam, Enass \\
\hline Bessière & Ugo & $\begin{array}{l}\text { "Quelle place pour les modèles } \\
\text { d'économie alternatifs dans l'Union } \\
\text { européenne? Ré-encastrer } \\
\text { l'économie dans la société ou quand } \\
\text { l'économie sociale et } \\
\text { l'entrepreneuriat social rencontrent } \\
\text { le marché intérieur" }\end{array}$ & $\begin{array}{l}\text { Master } \\
\text { Politique } \\
\text { et administration } \\
\text { européenne }\end{array}$ & $\begin{array}{l}\text { Collège d'Europe } \\
\text { Bruges }\end{array}$ \\
\hline Bloch & Baptiste & $\begin{array}{l}\text { "La CAE: limites, crise et mutations } \\
\text { d'un modèle économique » }\end{array}$ & Master & Lyon 2 \\
\hline Celle & Sylvain & $\begin{array}{l}\text { "Le mouvement coopératif } \\
\text { dans le socialisme de Jean Jaurès et } \\
\text { Marcel Mauss" }\end{array}$ & $\begin{array}{l}\text { Master } 1 \\
\text { Economie } \\
\text { appliquée }\end{array}$ & Lille 1 \\
\hline Diouf & $\begin{array}{l}\text { Jean-Noël } \\
\text { Diaga }\end{array}$ & $\begin{array}{l}\text { "Evaluation de la satisfaction des } \\
\text { clients en établissements } \\
\text { d'hébergement pour personnes } \\
\text { âgées dépendantes: le cas de } \\
\text { l'Ehpad du Beau Site de Clécy " }\end{array}$ & Master & $\begin{array}{l}\text { Lyon } 2 \text {, } \\
\text { Institut } \\
\text { de la francophonie } \\
\text { pour } \\
\text { l'administration } \\
\text { et la gestion }\end{array}$ \\
\hline Francoual & Pierre & $\begin{array}{l}\text { "Le travailleur dans la société } \\
\text { coopérative de production (Scop)» }\end{array}$ & $\begin{array}{l}\text { Master } 2 \\
\text { Droit du travail } \\
\text { et de l'emploi }\end{array}$ & Toulouse 1 \\
\hline Gautrey & Garance & $\begin{array}{l}\text { "La mise en place et l'internalisation } \\
\text { des pratiques transmises } \\
\text { aux producteurs dans le commerce } \\
\text { équitable: une approche néo- } \\
\text { institutionnaliste» }\end{array}$ & $\begin{array}{l}\text { Master } 2 \\
\text { Dynamique } \\
\text { des organisations, } \\
\text { travail } \\
\text { et ressources } \\
\text { humaines }\end{array}$ & Aix-Marseille \\
\hline Gayet & Franck & $\begin{array}{l}\text { "L'accompagnement à la mise } \\
\text { en coopération territoriale } \\
\text { des acteurs de l'ESS: l'enjeu } \\
\text { spécifique des organisations } \\
\text { sanitaires et sociales" }\end{array}$ & $\begin{array}{l}\text { Master } 1 \\
\text { Aménagement } \\
\text { territorial, } \\
\text { mémoire de stage }\end{array}$ & Lyon 3 \\
\hline
\end{tabular}




\begin{tabular}{|c|c|c|c|c|}
\hline Giordano & Florent & $\begin{array}{l}\text { "De la diffusion du discours } \\
\text { du new public management } \\
\text { vers l'éthique mutualiste» }\end{array}$ & $\begin{array}{l}\text { Master } 2 \\
\text { Recherche } \\
\text { en sciences } \\
\text { de gestion }\end{array}$ & $\begin{array}{l}\text { IAE Orléans- } \\
\text { Tours, Valorem }\end{array}$ \\
\hline Hartmann & Carine & $\begin{array}{l}\text { "Les mutations du secteur culturel: } \\
\text { vers une économie plus créative, } \\
\text { solidaire et durable» }\end{array}$ & $\begin{array}{l}\text { Master } 2 \\
\text { Ingénierie } \\
\text { de projets en ESS }\end{array}$ & Strasbourg \\
\hline $\begin{array}{l}\text { Lamy } \\
\text { de la } \\
\text { Chapelle }\end{array}$ & Albane & $\begin{array}{l}\text { "L'action collective des organisations } \\
\text { pour des agricultures alternatives: } \\
\text { de la pluralité des valeurs défendues } \\
\text { à l'élaboration d'un espace } \\
\text { de dialogue " }\end{array}$ & $\begin{array}{l}\text { Master } 2 \\
\text { Ressources } \\
\text { humaines ESS, } \\
\text { organisation } \\
\text { et projet }\end{array}$ & Méditerrannée \\
\hline Laze & Florian & $\begin{array}{l}\text { "Prémices d'un observatoire } \\
\text { social manceau » }\end{array}$ & $\begin{array}{l}\text { Master } 2 \\
\text { Management } \\
\text { et gestion } \\
\text { des entreprises de } \\
\text { l'économie sociale }\end{array}$ & Maine \\
\hline Lefebvre & Rémi & $\begin{array}{l}\text { "La NEF, coopérative financière } \\
\text { non banalisée » }\end{array}$ & $\begin{array}{l}\text { Master } 2 \\
\text { Développement } \\
\text { et expertise de } \\
\text { l'économie sociale }\end{array}$ & $\begin{array}{l}\text { Sciences } \\
\text { politiques } \\
\text { Grenoble }\end{array}$ \\
\hline Lerique & Florian & $\begin{array}{l}\text { "La préservation de l'image } \\
\text { de marque d'une structure } \\
\text { coopérative: entre } \\
\text { partage et contrôle, étude de cas } \\
\text { d'Enercoop» }\end{array}$ & $\begin{array}{l}\text { Master } \\
\text { Communication } \\
\text { des organisations }\end{array}$ & Paris XIII-Nord \\
\hline $\begin{array}{l}\text { Memdjofeng } \\
\text { Toche }\end{array}$ & $\begin{array}{l}\text { Christiane } \\
\text { Lolita }\end{array}$ & $\begin{array}{l}\text { "Les délocalisations des } \\
\text { entreprises et leurs répercussions } \\
\text { sur les Esat: le cas de l’Esat } \\
\text { de l'Association Marie-Pire» }\end{array}$ & $\begin{array}{l}\text { Master } 2 \\
\text { Entrepreneuriat des } \\
\text { projets socio- } \\
\text { économiques }\end{array}$ & $\begin{array}{l}\text { Lyon 2, Institut } \\
\text { de la francophonie } \\
\text { pour } \\
\text { l'administration } \\
\text { et la gestion }\end{array}$ \\
\hline Meyer & Camille & $\begin{array}{l}\text { "Les finances solidaires } \\
\text { comme biens communs durables: } \\
\text { étude de cas de la banque } \\
\text { communautaire de développement } \\
\text { Palmas (Brésil)» }\end{array}$ & $\begin{array}{l}\text { Master Sciences } \\
\text { de la population } \\
\text { et du } \\
\text { développement }\end{array}$ & $\begin{array}{l}\text { Université } \\
\text { libre } \\
\text { Bruxelles }\end{array}$ \\
\hline Moncel & Nathalie & $\begin{array}{l}\text { "L'habitat intergénérationnel: } \\
\text { une innovation sociale?» }\end{array}$ & $\begin{array}{l}\text { Master } \\
\text { Dynamique } \\
\text { des organisations, } \\
\text { travail et ressources } \\
\text { humaines }\end{array}$ & Aix-Marseille \\
\hline Pacalet & Max & $\begin{array}{l}\text { Etat, sport et cohésion sociale: } \\
\text { la coopération contractuelle } \\
\text { avec le mouvement sportif } \\
\text { constitue-t-elle un cadre adapté } \\
\text { pour mener une politique } \\
\text { d'inclusion sociale par le sport des } \\
\text { publics socialement défavorisés?» }\end{array}$ & $\begin{array}{l}\text { Master } 2 \\
\text { ESS }\end{array}$ & Lyon 2 \\
\hline
\end{tabular}




\begin{tabular}{|c|c|c|c|c|}
\hline Razanamandi & $\begin{array}{l}\text { Lalaina } \\
\text { Raymonde }\end{array}$ & $\begin{array}{l}\text { "Solidarité internationale } \\
\text { et co-développement, } \\
\text { les associations en France } \\
\text { œuvrant pour Madagascar: } \\
\text { le cas de Mitsinjo, } \\
\text { sept ans d'existence» }\end{array}$ & Master & $\begin{array}{l}\text { Paris } \\
\text { Oues-Nanterre }\end{array}$ \\
\hline Seeberger & Loïc & $\begin{array}{l}\text { "Essai sur le droit coopératif } \\
\text { français de ses origines } \\
\text { à la Ve République: entre } \\
\text { pratiques et normes juridiques » }\end{array}$ & $\begin{array}{l}\text { Master } 2 \\
\text { Histoire du droit }\end{array}$ & Montpellier 1 \\
\hline Tabet & Alexei & $\begin{array}{l}\text { "L'invention du “tiers secteur”: } \\
\text { les commencements de l'ESS" }\end{array}$ & $\begin{array}{l}\text { Master } 2 \\
\text { Histoire }\end{array}$ & IEP Paris \\
\hline Tarantini & Guillaume & $\begin{array}{l}\text { «Les banques coopératives } \\
\text { face à la crise financière } \\
\text { et économique: l'émergence } \\
\text { d'un nouveau business model?» }\end{array}$ & $\begin{array}{l}\text { Master } 2 \\
\text { ESS }\end{array}$ & Lumière-Lyon 2 \\
\hline Zellal & Ahlem & $\begin{array}{l}\text { "Approche théorique } \\
\text { de la gestion des connaissances } \\
\text { et des apprentissages } \\
\text { organisationnels dans un système } \\
\text { coopétitif, facteurs favorisant } \\
\text { la collaboration et le partage» }\end{array}$ & $\begin{array}{l}\text { Master } 2 \\
\text { recherche } \\
\text { - Stratégie } \\
\text { et management }\end{array}$ & $\begin{array}{l}\text { Université } \\
\text { Paris XI-Sud }\end{array}$ \\
\hline AZouatcham & $\begin{array}{l}\text { Hubert- } \\
\text { Patrice }\end{array}$ & $\begin{array}{l}\text { "Les principes coopératifs } \\
\text { dans la société coopérative } \\
\text { d'épargne et de crédit Ohada » }\end{array}$ & $\begin{array}{l}\text { Master } 2 \\
\text { Recherche droit } \\
\text { économique }\end{array}$ & $\begin{array}{l}\text { Nice-Sophia } \\
\text { Antipolis }\end{array}$ \\
\hline
\end{tabular}

